\title{
Factors influencing completion of multi- dose vaccine schedules in adolescents: a systematic review
}

\author{
K. E. Gallagher ${ }^{1,2^{*}}$, E. Kadokura ${ }^{3}$, L. O. Eckert ${ }^{4}$, S. Miyake' ${ }^{1}$ S. Mounier-Jack ${ }^{5}$, M. Aldea ${ }^{6,7}$, D. A. Ross ${ }^{8}$ \\ and D. Watson-Jones ${ }^{1,2}$
}

\begin{abstract}
Background: Completion of multiple dose vaccine schedules is crucial to ensure a protective immune response, and maximise vaccine cost-effectiveness. While barriers and facilitators to vaccine uptake have recently been reviewed, there is no comprehensive review of factors influencing subsequent adherence or completion, which is key to achieving vaccine effectiveness. This study identifies and summarises the literature on factors affecting completion of multi-dose vaccine schedules by adolescents.
\end{abstract}

Methods: Ten online databases and four websites were searched (February 2014). Studies with analysis of factors predicting completion of multi-dose vaccines were included. Study participants within 9-19 years of age were included in the review. The defined outcome was completion of the vaccine series within 1 year among those who received the first dose.

Results: Overall, 6159 abstracts were screened, and 502 full texts were reviewed. Sixty one studies were eligible for this review. All except two were set in high-income countries. Included studies evaluated human papillomavirus vaccine, hepatitis $A$, hepatitis $B$, and varicella vaccines. Reported vaccine completion rates, among those who initiated vaccination, ranged from $27 \%$ to over $90 \%$. Minority racial or ethnic groups and inadequate health insurance coverage were risk factors for low completion, irrespective of initiation rates. Parental healthcare seeking behaviour was positively associated with completion. Vaccine delivery in schools was associated with higher completion than delivery in the community or health facilities. Gender, prior healthcare use and socio-economic status rarely remained significant risks or protective factors in multivariate analysis.

Conclusions: Almost all studies investigating factors affecting completion have been carried out in developed countries and investigate a limited range of variables. Increased understanding of barriers to completion in adolescents will be invaluable to future new vaccine introductions and the further development of an adolescent health platform. PROSPERO reg\# CRD42014006765.

Keywords: Vaccines and immunization, Immunization programmes, Vaccination completion, Barriers, Adolescent health services

\footnotetext{
* Correspondence: Katherine.Gallagher@lshtm.ac.uk

${ }^{1}$ Clinical Research Department, Faculty of Infectious Tropical Diseases,

London School of Hygiene and Tropical Medicine, Keppel Street, London

WC1E 7HT, UK

${ }^{2}$ Mwanza Intervention Trials Unit, The National Institute for Medical Research

Mwanza Campus, PO Box 11936, Mwanza, Tanzania

Full list of author information is available at the end of the article
} 


\section{Background}

In the past decade there has been an increase in the number of new vaccines licensed worldwide $[1,2]$ and in the accessible funding for vaccine introduction to lowresource settings through the founding of Gavi, The Vaccine Alliance, in 2000 [3]. Multi-dose vaccines in the WHO recommended immunization schedule for adolescents are listed in Table 1; WHO defines adolescence as age 10-19 years inclusive. National vaccine schedules can depart from WHO recommendations, e.g. 2 doses of hepatitis A and meningococcal conjugate vaccines (MCV4) are offered to adolescents in the USA [4]. Although recommended for administration at birth, hepatitis $B$ vaccine $(\mathrm{HBV})$ is routinely offered to older children and adolescents if not previously immunised [5]. In settings where varicella is seen as a public health priority WHO recommends 2 doses of varicella vaccine, with the first dose at 12-18 months and up to 4 month interval between doses $[6,7]$. The most recently licensed multidose vaccines are the human papillomavirus (HPV) vaccines. In 2014, HPV vaccine recommendations were revised by WHO SAGE from a schedule of 3 doses [8], to 2 doses at a 6 month interval in girls less than or equal to 15 years of age $[9,10]$ based on evidence of non-inferior immunogenicity $[11,12]$.
At present, evidence suggests multiple doses of HBV, $\mathrm{HPV}$, and varicella vaccines are needed for efficacious protection against disease in adolescents [5, 9, 13, 14]. However; completion of the vaccine dose series, defined as receipt of the final dose within 1 year of the first dose, has proven challenging in some settings. Completion rates of HPV vaccine were lower than $30 \%$ in the first years of introduction in the USA $[15,16]$. Addressing specific difficulties in administering vaccines to adolescents will be invaluable for implementation of future adolescent vaccines and further developing adolescent health services.

The currently available reviews of factors influencing completion focus solely on selected developed countries [1, 17-19], have non-systematic searches [20, 21] or need updating [22]. This systematic review describes factors which have been investigated for their effect on multi-dose vaccine adherence in adolescents to aid development of interventions to improve adherence.

\section{Methods \\ Search strategy}

A comprehensive set of search terms was built around:

1) childhood/adolescence; 2) vaccination/immunisation;

3) adherence/completion. Articles with at least one term

Table 1 WHO recommended vaccine schedule for adolescents ${ }^{\mathrm{a}, \mathrm{b}}$. Caption: the WHO recommended schedule of vaccines for adolescents (10-19 years of age inclusive), if not given prior to age 10 years

\begin{tabular}{|c|c|c|}
\hline Recommended vaccines for all adolescents & Adolescent dosage & Licensed age \\
\hline Tetanus, diphtheria, pertussis & 3 doses Tdap ${ }^{a} \&$ Td Booster & Infant onwards \\
\hline \multirow[t]{2}{*}{ Human papillomavirus } & 2 doses if $\leq 15$ years & $\geq 9$ years \\
\hline & 3 doses thereafter & \\
\hline \multirow[t]{3}{*}{ Meningococcal conjugate } & MenA: 1 dose & Infant onwards \\
\hline & MenC: 1 dose & \\
\hline & MCV4: 1 dose & \\
\hline Influenza & 1 dose Yearly booster & $\geq 9$ years \\
\hline Hepatitis A & 1 dose & Infant onwards \\
\hline Hepatitis B & 3 doses $^{\mathrm{a}}$ & Infant onwards \\
\hline Measles, Mumps, Rubella & 1 dose $^{a}$ & Infant onwards \\
\hline Recommended in at-risk areas & Adolescent dosage & Licensed age \\
\hline Tick borne encephalitis & 3 doses & Infant onwards \\
\hline Japanese encephalitis & 1 dose & Infant onwards \\
\hline \multirow[t]{3}{*}{ Typhoid } & Vi polysaccharide: 1 dose & Infant onwards \\
\hline & Ty21a live oral vaccine: $3-4$ doses & \\
\hline & Booster 3-7 years after primary series & \\
\hline Cholera & Dukoral, Shanchol \& mORCVAX: 2 doses booster every $2^{\text {nd }}$ yr & $\geq 2$ years \\
\hline Rabies & 3 doses & Infant onwards \\
\hline Varicella & 2 doses & $\geq 9-12$ months \\
\hline
\end{tabular}

${ }^{\mathrm{a}}$ Recommended schedule if not administered prior to age 10 years

${ }^{b}$ World Health Organization. WHO recommendations for routine immunization - summary tables -

http://www.who.int/immunization/policy/immunization_tables/en/ 2014 
from each topic were identified. Search terms were informed by the Cochrane Child Health Group recommended terms for adolescents or school children [23] and included international spelling variations (Additional file 1: Table S1). Multi-dose vaccines administered to adolescents were identified through the Centers for Disease, Control, and Prevention (CDC) [6, 24], and the WHO list of prequalified vaccines [25]; however, search terms were not limited to these vaccines.

Medline, Embase, Global Health (Ovid SP), Popline, Web of Science, Africa Portal, Africa-wide information, ADOLEC, Cochrane, Open Grey databases, and PATH, Gavi, and WHO websites were searched in February 2014. No publication date restriction was set. Publications, abstracts and conference proceeding were eligible for inclusion. All texts were collated and reviewed using Endnote X7 (Thompson Reuters); automated and manual de-duplication was performed.

\section{Inclusion criteria}

Inclusion criteria for consideration of studies were outlined in a protocol a-priori as per PRISMA guidelines [26] (Table 2). The title and/or abstract of each article were reviewed in the first instance by a single reviewer (KG). Modelling studies, immunogenicity/efficacy trials were excluded. Two reviewers (KG, SM/EK) screened the abstracts. Any study including a vaccine for which more than one dose was administered to persons 9-19 years old in a routine setting within 1 year was considered for inclusion. The WHO definition of adolescent (10-19 years old) was widened to include 9 year olds to include all participants in HPV vaccine studies (WHO recommended for 9-13 year olds). Inclusion criteria were independently applied to full texts by 2 authors (KG, SM/EK) (Fig. 1) [26].

\section{Data extraction}

Data were extracted by 2 authors (KG, EK) into separate forms on Microsoft Excel 2010. Article selection and data extraction discrepancies were resolved through discussion. Data on the study population, setting, vaccine, rates of completion and the factors investigated were extracted alongside descriptive, univariate and multivariate results, as applicable.

\section{Assessment of bias}

An assessment of bias was recorded on the data extraction form for each study using criteria outlined in the Cochrane tool for assessment of bias in intervention and epidemiological studies [27]. Selection bias and information bias were assessed alongside the potential for confounding.

\section{Data synthesis}

Heterogeneity in study methods, population, context and classification of exposure variables, led to a descriptive synthesis. Groups which initiated and completed the recommended vaccine schedule within 1 year were compared to non-completers who only initiated.

\section{Results}

Of the 502 full texts reviewed (Fig. 1), 61 articles were eligible for inclusion (Table 3). Included articles reported completion rates for HPV (3-dose completion ranged from $27 \%$ [15] to over $90 \%$ [28]), HBV (27\% completion before a school mandate was introduced in California [29] to $95 \%$ in a school-based programme in Canada [30]),

Table 2 Study inclusion criteria. Caption: abstracts and full texts were screened independently by two authors using the following criteria

\begin{tabular}{ll}
$\begin{array}{l}\text { Study definitions and } \\
\text { characteristics }\end{array}$ & Inclusion criteria: studies investigating factors governing adherence \\
\hline Study population & $\begin{array}{l}\text { Any child/adolescent 9-19 years old, recruited from the community or a cohort of vaccinees, care-givers or care- } \\
\text { providers }\end{array}$ \\
Geographical setting & $\begin{array}{l}\text { No restriction } \\
\text { Any vaccine administered to the study population in a schedule including more than one } \\
\text { dose within the same year }\end{array}$ \\
Vaccine delivery & $\begin{array}{l}\text { Routine vaccine delivery; studies excluded if an outbreak/campaign setting/non-routine delivery } \\
\text { Cutcome }\end{array}$ \\
within 1 year of follow-up
\end{tabular}




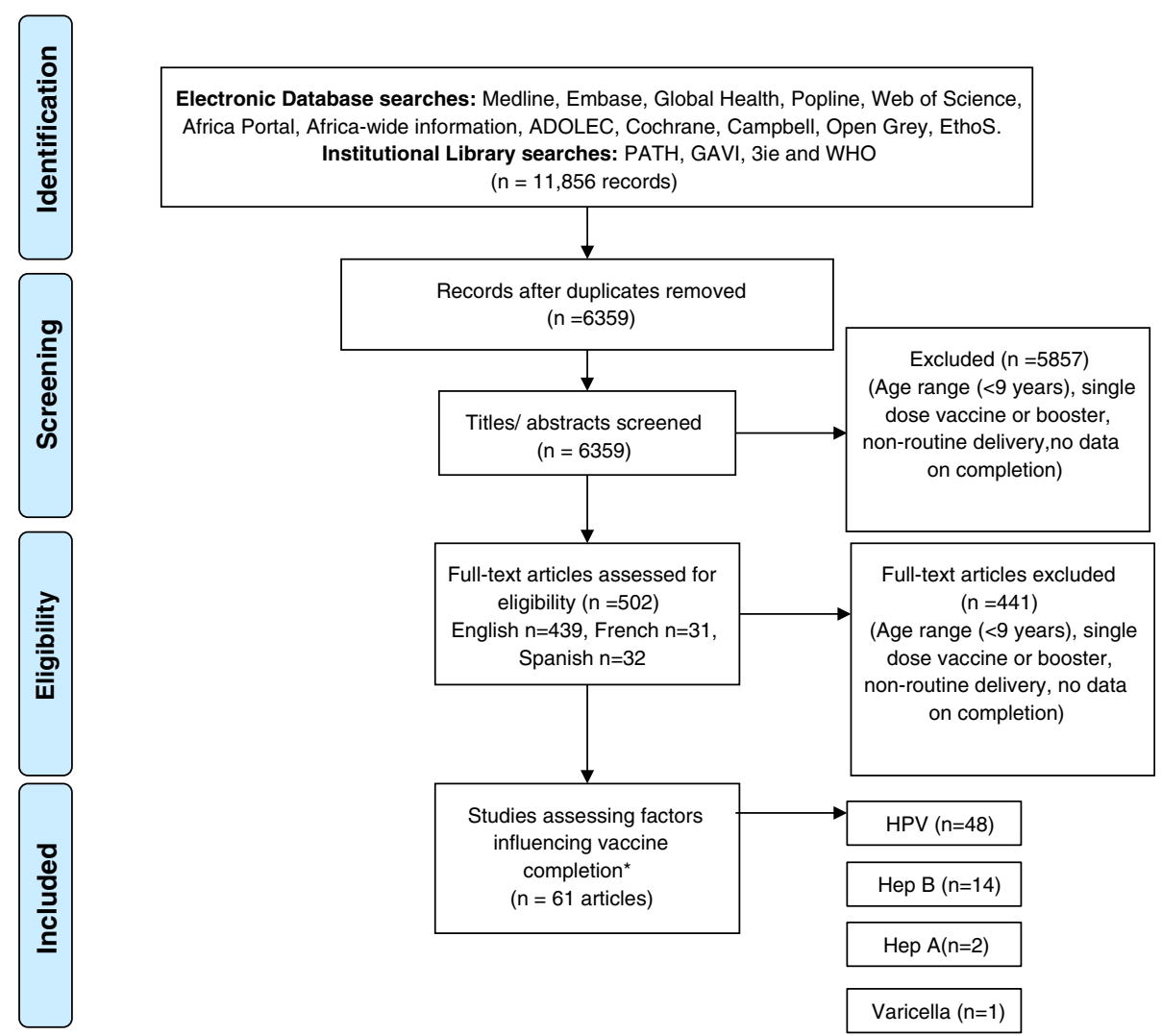

Fig. 1 Systematic review flow diagram. Caption: the PRISMA flow diagram for the systematic review detailing the database searches, the number of abstracts screened and the full texts retrieved. ${ }^{*}$ Some articles analysed $>1$ vaccine

varicella and hepatitis A vaccine (HAV). In the USA, the two dose series of varicella vaccine was completed within 1 year in $35.9 \%$ of adolescents and 2 doses of HAV were completed in 40-48 \% [31]. Searches returned no articles on completion of conjugate meningococcal vaccine, despite a multi-dose policy in the USA [32]. For the purposes of this review we have focused on results from multivariate analyses or qualitative findings. Data availability by country and vaccine is displayed in Table 4 .

\section{Individual level factors}

Age

The association between age and completion was investigated in 31 articles. Multivariate analyses of at least 2 age categories within the age range of 9-19 years were conducted in 20 studies, in the USA $(n=17)$, Canada [33], France [34], and Australia [35]. Age recommendations vary across countries; results must be interpreted in context.

There is some evidence that completion rates decrease as age of vaccine initiation increases for HPV vaccine, HAV, and HBV $[15,31,36-38]$. In the USA, the HPV vaccine recommended age range is between 11 and 26 years; five studies state similar results among Medicaid enrolees, adjusting for insurance, race, region and year, 17 year olds were 0.84 times less likely to complete HPV vaccine compared to 11 year olds (95\% CI 0.74-0.95) [15]. Among attendees of an urban hospital, in adjusted analyses, 14-17 year olds had 0.71 the odds of completion HPV vaccine when compared to 9-13 years olds (95\% CI 0.59-0.98) [37].

In the USA five further studies found no association $[33,39-43]$ and two studies report the converse association, increased likelihood of completion with age between 13 and 17 years controlling for year, race, insurance status; this perhaps reflects the perception that it was an 'STI vaccine' in 2007-8 [44, 45]. No association between age and HPV vaccine completion was found in multivariate analyses in Canada although only one school grade was targeted [33, 39-43].

\section{Race}

Racial or ethnic identity was analysed in 31 studies from the USA, Australia and Greece; 18 conducted multivariate analyses. Analysis of $>100,000$ women in North Carolina adjusted for location, clinic, insurance, and age found Black (aOR 0.55; 95 \% CI 0.53-0.56), American Indian or Alaskan (aOR 0.68; 0.61-0.77) and Hispanic 
Table 3 Summary of included studies. Caption: a summary of the studies included in the review, including details of sample size, the source of the sample, year of data collection, vaccine investigated, target age group, delivery strategy, completion rate attained and factors investigated to influence completion

\begin{tabular}{|c|c|c|c|c|c|c|c|c|}
\hline Author; date & Sample size & Country; source of sample & $\begin{array}{l}\text { Year of data } \\
\text { collection }\end{array}$ & Vaccine & $\begin{array}{l}\text { Vaccine target } \\
\text { age group }\end{array}$ & $\begin{array}{l}\text { Vaccine delivery } \\
\text { strategy }\end{array}$ & Completion rate & $\begin{array}{l}\text { Factors investigated to } \\
\text { influence completion }\end{array}$ \\
\hline $\begin{array}{l}\text { Bednarczyk, R; } \\
\text { et al. } 2011\end{array}$ & 588 & $\begin{array}{l}\text { USA. New York state } \\
\text { University health clinics } \\
\text { and classrooms: self-report } \\
\text { questionnaire. }\end{array}$ & 2010 & HPV & 11-12 (with catch up to 26) & $\begin{array}{l}\text { Available at routine } \\
\text { healthcare provider }\end{array}$ & $79 \%$ & Qualitative interviews \\
\hline $\begin{array}{l}\text { Carlos, R; } \\
\text { et al. } 2010\end{array}$ & 232 & $\begin{array}{l}\text { USA. Mailed questionnaire } \\
\text { to attendees of breast and } \\
\text { cervical cancer screening } \\
\text { clinics (maternal report). }\end{array}$ & 2010 & HPV & 11-12 (with catch up to 26) & $\begin{array}{l}\text { Available at routine } \\
\text { healthcare provider }\end{array}$ & $19 \%$ & Race \\
\hline $\begin{array}{l}\text { Cassidy, W; } \\
\text { Mahoney, F. } 1995\end{array}$ & 654 & $\begin{array}{l}\text { USA. School and } \\
\text { administrative data. }\end{array}$ & $1994-5$ & HBV & School grades 6-8 & School-based clinic & $82 \%$ & Gender, race \\
\hline $\begin{array}{l}\text { Chao, C; Slezak, } \\
\text { J; et al. } 2009\end{array}$ & 18,275 & $\begin{array}{l}\text { USA. Electronic health } \\
\text { records from KPSC managed } \\
\text { care organisation. }\end{array}$ & $2006-8$ & HPV & $9-26$ & $\begin{array}{l}\text { Available at routine } \\
\text { healthcare provider }\end{array}$ & $43 \%$ & $\begin{array}{l}\text { Maternal characteristics: } \\
\text { history of at least } 1 \text { Pap } \\
\text { test in the past } 3 \text { years, } \\
\text { history of abnormal pap } \\
\text { test result, history of genital } \\
\text { warts/ other STIs, SES } \\
\text { (neighbourhood median } \\
\text { household income, } \\
\text { neighbourhood average } \\
\text { adult education) }\end{array}$ \\
\hline $\begin{array}{l}\text { Chao, C; Velicer, } \\
\text { C; et al. } 2009\end{array}$ & 34,193 & $\begin{array}{l}\text { USA. Electronic health } \\
\text { records from KPSC } \\
\text { managed care organisation. }\end{array}$ & $2006-8$ & HPV & $9-26$ & $\begin{array}{l}\text { Available at routine } \\
\text { healthcare provider }\end{array}$ & $41 \%$ & $\begin{array}{l}\text { age, race, socioeconomic } \\
\text { status (census block } \\
\text { neighbourhood statistics, } \\
\text { medicaid eligibility), provider } \\
\text { characteristics, health care } \\
\text { utilization, women's health } \\
\text { related conditions, chronic } \\
\text { illness }\end{array}$ \\
\hline $\begin{array}{l}\text { Chou, B; } \\
\text { et al. } 2011\end{array}$ & 1413 & $\begin{array}{l}\text { USA. Electronic health } \\
\text { records from ambulatory } \\
\text { care clinics (4) associated } \\
\text { with a University. }\end{array}$ & $2007-8$ & HPV & 11-12 (with catch up to 26) & $\begin{array}{l}\text { Available at routine } \\
\text { healthcare provider }\end{array}$ & $33 \%$ & $\begin{array}{l}\text { Age, insurance (private/public), } \\
\text { provider characteristics } \\
\text { (location, practice type } \\
\text { (pediatrics, gynaecology or } \\
\text { family practice)), race (White, } \\
\text { African American, Hispanic). }\end{array}$ \\
\hline Cleves, M. 1998 & 520 & USA. Medical records. & $1995-6$ & HBV & $11-19$ & $\begin{array}{l}\text { Available at routine } \\
\text { healthcare provider }\end{array}$ & $33 \%$ & $\begin{array}{l}\text { Age, race, insurance, sexual } \\
\text { activity, risk behaviour } \\
\text { (drug use) }\end{array}$ \\
\hline $\begin{array}{l}\text { Cook, R; } \\
\text { et al. } 2010\end{array}$ & 11,986 & $\begin{array}{l}\text { USA. Medicaid } \\
\text { administrative data. }\end{array}$ & $2006-8$ & HPV & $9-20$ & $\begin{array}{l}\text { Available at routine } \\
\text { healthcare provider }\end{array}$ & $27 \%$ & $\begin{array}{l}\text { Age, race, provider of first } \\
\text { shot, insurance (months of } \\
\text { medicaid enrollment), } \\
\text { sexual activity. }\end{array}$ \\
\hline
\end{tabular}


Table 3 Summary of included studies. Caption: a summary of the studies included in the review, including details of sample size, the source of the sample, year of data collection, vaccine investigated, target age group, delivery strategy, completion rate attained and factors investigated to influence completion (Continued)

\begin{tabular}{|c|c|c|c|c|c|c|c|c|}
\hline \multirow{2}{*}{$\begin{array}{l}\text { Crosby, R; } \\
\text { et al. } 2011\end{array}$} & \multirow[t]{2}{*}{209} & \multirow{2}{*}{$\begin{array}{l}\text { USA. University of Kentucky, } \\
\text { rural community college } \\
\text { and rural health clinic } \\
\text { attendees }\end{array}$} & \multirow[t]{2}{*}{$2007-8$} & \multirow[t]{2}{*}{$\mathrm{HPV}$} & \multirow[t]{2}{*}{$9-26$} & \multirow{2}{*}{$\begin{array}{l}\text { Available at routine } \\
\text { healthcare provider }\end{array}$} & $56 \%$ urban & \multirow{2}{*}{$\begin{array}{l}\text { Geography (rural/urban } \\
\text { location) }\end{array}$} \\
\hline & & & & & & & $10 \%$ rural & \\
\hline $\begin{array}{l}\text { Deeks, S; } \\
\text { Johnson, } \\
\text { I. } 1998\end{array}$ & 39,935 & $\begin{array}{l}\text { Canada. Administrative } \\
\text { data from Health units (7), } \\
\text { Greater Toronto Area }\end{array}$ & $1994-5$ & HBV & School Grade 7 & $\begin{array}{l}\text { School-based } \\
\text { delivery }\end{array}$ & $95 \%$ & $\begin{array}{l}\text { School characteristics, } \\
\text { knowledge/ education/ } \\
\text { mobilisation }\end{array}$ \\
\hline $\begin{array}{l}\text { Dempsey, A; } \\
\text { et al. } 2010\end{array}$ & 2625 & $\begin{array}{l}\text { USA. Health records from } \\
20 \text { university-affiliated } \\
\text { health clinics, Michigan }\end{array}$ & $2007-8$ & HPV & $9-26$ & $\begin{array}{l}\text { Available at routine } \\
\text { healthcare provider }\end{array}$ & $15 \%$ & Age, insurance, race \\
\hline $\begin{array}{l}\text { Dempsey, A; } \\
\text { et al. } 2012\end{array}$ & 1714 & $\begin{array}{l}\text { USA. Health records from } \\
20 \text { university-affiliated } \\
\text { health clinics, Michigan }\end{array}$ & $2008-9$ & HPV & $9-26$ & $\begin{array}{l}\text { Available at routine } \\
\text { healthcare provider }\end{array}$ & $53 \%$ & Age, insurance, race \\
\hline $\begin{array}{l}\text { Dorell, } C_{i} \\
\text { et al. } 2011\end{array}$ & 18,228 & $\begin{array}{l}\text { USA. Stratified, national, } \\
\text { probability sample of } \\
\text { households (NIS-teen } \\
\text { survey) }\end{array}$ & $2008-10$ & HPV & $9-26$ & $\begin{array}{l}\text { Available at routine } \\
\text { healthcare provider }\end{array}$ & $53 \%$ & $\begin{array}{l}\text { Age, insurance, health care } \\
\text { utilization, household income, } \\
\text { maternal education level, } \\
\text { maternal age, maternal marital } \\
\text { status, race, geography/ } \\
\text { location }\end{array}$ \\
\hline $\begin{array}{l}\text { Fournier, M; } \\
\text { et al. } 2013\end{array}$ & 1404 & $\begin{array}{l}\text { USA. Electronic medical } \\
\text { records from } 2 \text { primary } \\
\text { care clinics }\end{array}$ & $2007-12$ & HPV & 11-12 (catch up to 26) & $\begin{array}{l}\text { Available at routine } \\
\text { healthcare provider }\end{array}$ & $40 \%$ & $\begin{array}{l}\text { Insurance, race, health care } \\
\text { utilization (other vaccines) }\end{array}$ \\
\hline \multirow{2}{*}{$\begin{array}{l}\text { Ganry, O; } \\
\text { et al. } 2013\end{array}$} & & \multirow{2}{*}{$\begin{array}{l}\text { France. Electronic records } \\
\text { of the Regime General } \\
\text { Insurance (for workers), } \\
\text { the RSI (for self-employed) } \\
\text { and the RSA (agricultural } \\
\text { occupations) }\end{array}$} & \multirow[t]{2}{*}{ 2009-10 } & \multirow[t]{2}{*}{ HPV } & 14 (with a catch up to 23) & \multirow{2}{*}{$\begin{array}{l}\text { Available at routine } \\
\text { healthcare provider }\end{array}$} & \multirow[t]{2}{*}{$39 \%$} & \multirow{2}{*}{$\begin{array}{l}\text { Age, insurance, provider } \\
\text { characteristics. }\end{array}$} \\
\hline & & & & & (recently revised to 11-14) & & & \\
\hline $\begin{array}{l}\text { Gold, R; } \\
\text { et al. } 2013\end{array}$ & 786 & $\begin{array}{l}\text { USA. Electronic medical } \\
\text { records from an integrated } \\
\text { managed care organisation }\end{array}$ & 2008 & HPV & 11-12 (catch up to 26) & $\begin{array}{l}\text { Available at routine } \\
\text { healthcare provider }\end{array}$ & & $\begin{array}{l}\text { Socioeconomic status; health } \\
\text { care utilization; provider } \\
\text { characteristics; vaccine delivery } \\
\text { concomitant with first dose; } \\
\text { experiences at the first visit, } \\
\text { challenges to making or } \\
\text { keeping the index } \\
\text { appointment; Knowledge } \\
\text { and attitudes about HPV; } \\
\text { adverse events. }\end{array}$ \\
\hline $\begin{array}{l}\text { Gold, R; } \\
\text { et al. } 2011\end{array}$ & 450 & $\begin{array}{l}\text { USA. Administrative data } \\
\text { from } 19 \text { school-based } \\
\text { health centres }\end{array}$ & $2007-8$ & HPV & 11-12 (catch up to 26) & $\begin{array}{l}\text { Available at routine } \\
\text { healthcare provider }\end{array}$ & $51 \%$ & Age, race and insurance status \\
\hline $\begin{array}{l}\text { Gonzalez, l; } \\
\text { et al. } 2002\end{array}$ & 79,357 & $\begin{array}{l}\text { USA. Electronic Data from } \\
3 \text { Health Management } \\
\text { Organisations }\end{array}$ & 1998 & HBV & $11-12$ & $\begin{array}{l}\text { Available at routine } \\
\text { healthcare provider }\end{array}$ & $73 \%, 67 \%, 45 \%$ & $\begin{array}{l}\text { Provider characteristics } \\
\text { (Health care organisation) }\end{array}$ \\
\hline
\end{tabular}


Table 3 Summary of included studies. Caption: a summary of the studies included in the review, including details of sample size, the source of the sample, year of data collection, vaccine investigated, target age group, delivery strategy, completion rate attained and factors investigated to influence completion (Continued)

\begin{tabular}{|c|c|c|c|c|c|c|c|c|}
\hline $\begin{array}{l}\text { Harper, D; } \\
\text { et al. } 2013\end{array}$ & 2422 & $\begin{array}{l}\text { USA. Electronic records } \\
\text { from a safety net health } \\
\text { care system Kansas }\end{array}$ & 2006-09 & HPV & $11-12$ (catch up to 26) & $\begin{array}{l}\text { Available at routine } \\
\text { healthcare provider }\end{array}$ & $42 \%$ & $\begin{array}{l}\text { Age, race, concomitant (visit } \\
\text { type for first dose) }\end{array}$ \\
\hline $\begin{array}{l}\text { Hirth, J; } \\
\text { et al. } 2012\end{array}$ & 271,976 & $\begin{array}{l}\text { USA. Electronic records of a } \\
\text { private insurance company }\end{array}$ & $2006-10$ & HPV & 11-12 (catch up to 26) & $\begin{array}{l}\text { Available at routine } \\
\text { healthcare provider }\end{array}$ & $38 \%$ & Age, provider type, time \\
\hline $\begin{array}{l}\text { Kester, L; } \\
\text { et al. } 2011\end{array}$ & 500 & $\begin{array}{l}\text { USA. Knowledge networks } \\
\text { coordinated survey } \\
\text { (nationally representative) }\end{array}$ & 2010 & HPV & 11-12 (catch up to 26) & $\begin{array}{l}\text { Available at routine } \\
\text { healthcare provider }\end{array}$ & $81 \%$ & $\begin{array}{l}\text { Race, insurance, maternal } \\
\text { education, maternal } \\
\text { relationship status, maternal } \\
\text { history of HPV related condition, } \\
\text { geography. }\end{array}$ \\
\hline $\begin{array}{l}\text { Kouyoumdjian, } \\
\text { F; Bailowitz, } \\
\text { A. } 2011\end{array}$ & 18 & $\begin{array}{l}\text { USA. Baltimore city health } \\
\text { department self report } \\
\text { interviews }\end{array}$ & $2007-9$ & HPV & 11-12 (catch up to 26) & $\begin{array}{l}\text { Available at routine } \\
\text { healthcare provider }\end{array}$ & $9.80 \%$ & $\begin{array}{l}\text { Geography (access), adverse } \\
\text { events, qualitative reasons } \\
\text { (convenience, knowledge, } \\
\text { pain/discomfort) }\end{array}$ \\
\hline \multirow[t]{4}{*}{$\begin{array}{l}\text { Ladner, J; } \\
\text { et al. } 2012\end{array}$} & \multirow[t]{4}{*}{87580} & \multirow{4}{*}{$\begin{array}{l}\text { Multi-country ( } 7 \text { low } \\
\text { resource countries). } \\
\text { Administrative data from } \\
\text { Gardasil Access Programme } \\
\text { grantee countries }\end{array}$} & \multirow[t]{4}{*}{ 2009-11 } & \multirow[t]{4}{*}{ HPV } & $\begin{array}{l}\text { Bhutan, Bolivia, Haiti, } \\
\text { Nepal: 9-13 }\end{array}$ & \multirow{4}{*}{$\begin{array}{l}\text { School based, health } \\
\text { centre based or } \\
\text { mixed strategies }\end{array}$} & \multirow{4}{*}{$\begin{array}{l}\text { Bhutan: } 88 \% \text {, Bolivia: } \\
96 \% \text {; Cambodia: } 95 \% \text {; } \\
\text { Cameroon } 83 \% \text {, Haiti } 76 \% \text {, } \\
\text { Lesotho } 93 \% \text {; } \\
\text { Nepal } 99 \% \text {. }\end{array}$} & \multirow{4}{*}{$\begin{array}{l}\text { Delivery strategy (school- } \\
\text { based, health facility model, } \\
\text { mixed model) }\end{array}$} \\
\hline & & & & & Cambodia: 11-18 & & & \\
\hline & & & & & Cameroon: 9-18 & & & \\
\hline & & & & & Lesotho: 10-18 & & & \\
\hline $\begin{array}{l}\text { Lancman, } \mathrm{H}_{\text {; }} \\
\text { et al. } 2000\end{array}$ & 3 centres & $\begin{array}{l}\text { USA. Administrative data } \\
\text { from } 2 \text { school based health } \\
\text { centres and one adolescent } \\
\text { health clinic }\end{array}$ & 1997-98 & HBV & 11 and above & $\begin{array}{l}\text { Available at school } \\
\text { based health centres } \\
\text { and routine providers }\end{array}$ & $24 \%, 29 \%, 76 \%$ & Health centre characteristics \\
\hline $\begin{array}{l}\text { Laz, T; } \\
\text { et al. } 2012\end{array}$ & 11,277 & $\begin{array}{l}\text { USA. Household } \\
\text { questionnaire sent to } \\
\text { parents }\end{array}$ & 2010 & HPV & 11-12 (catch up to 26) & $\begin{array}{l}\text { Available at routine } \\
\text { healthcare provider }\end{array}$ & $49 \%$ & $\begin{array}{l}\text { Age, parental education, } \\
\text { insurance, race, parental } \\
\text { income. }\end{array}$ \\
\hline $\begin{array}{l}\text { Lions, } C_{i} \\
\text { et al. } 2013\end{array}$ & 105,327 & $\begin{array}{l}\text { France. National Insurance } \\
\text { Reimbursement database }\end{array}$ & $2007-8$ & HPV & 11-14 (catch up to 19) & $\begin{array}{l}\text { Available at routine } \\
\text { healthcare provider }\end{array}$ & $64.10 \%$ & $\begin{array}{l}\text { Age, insurance, geography, } \\
\text { medical utilization }\end{array}$ \\
\hline $\begin{array}{l}\text { Macdonald, } \\
\text { V; et al. } 2007\end{array}$ & 2471 & $\begin{array}{l}\text { Australia. Health centre } \\
\text { records from a primary } \\
\text { health care centre, Sydney } \\
\text { (high risk population) }\end{array}$ & $1992-2003$ & HBV & $\begin{array}{l}\text { High risk adolescent } \\
\text { of any age }\end{array}$ & $\begin{array}{l}\text { Available at routine } \\
\text { healthcare provider } \\
\text { and specialist clinics }\end{array}$ & $21 \%$ & $\begin{array}{l}\text { Age, gender, race (aboriginal), } \\
\text { risk behaviour (IDU, sex worker, } \\
\text { hep.C status) length of contact } \\
\text { with the health centre, } \\
\text { accelerated versus normal } \\
\text { schedule. }\end{array}$ \\
\hline $\begin{array}{l}\text { Moore, G; } \\
\text { et al. } 2010\end{array}$ & 209 & $\begin{array}{l}\text { USA. Medical records of } \\
\text { community health clinic } \\
\text { attendees }\end{array}$ & Unknown & HPV & $11-12$ (catch up to 26) & $\begin{array}{l}\text { Available at routine } \\
\text { healthcare provider }\end{array}$ & $28 \%$ & $\begin{array}{l}\text { Attitudes and believes } \\
\text { including perceptions of } \\
\text { risk, peer experience of HPV } \\
\text { vaccine, experience of cancer }\end{array}$ \\
\hline
\end{tabular}


Table 3 Summary of included studies. Caption: a summary of the studies included in the review, including details of sample size, the source of the sample, year of data collection, vaccine investigated, target age group, delivery strategy, completion rate attained and factors investigated to influence completion (Continued)

\begin{tabular}{|c|c|c|c|c|c|c|c|c|}
\hline $\begin{array}{l}\text { Nelson, J C; } \\
\text { et al; } 2009\end{array}$ & 590445 & $\begin{array}{l}\text { USA. Vaccine Safety } \\
\text { Datalink population (MCO } \\
\text { registry) }\end{array}$ & $1996-2004$ & $\begin{array}{l}\text { Varicella, } \\
\text { HAV, } \\
\text { HBV }\end{array}$ & $9-17$ & $\begin{array}{l}\text { Available at routine } \\
\text { healthcare provider }\end{array}$ & $\begin{array}{l}\text { Varicella: } 35.9 \% \text { \% HAV: } \\
48.4 \% \text { (age 9-12), } 40.3 \% \\
\text { (age 13-17); HBV: } 63.4 \% \\
\text { (age 9-12), 45.1 \% (age } \\
\text { 13-17). }\end{array}$ & $\begin{array}{l}\text { Age, provider site, gender, } \\
\text { length of MCO enrollment, } \\
\text { year of first dose, utilization of } \\
\text { medical visits in year prior to } \\
\text { dose } 1\end{array}$ \\
\hline \multirow{3}{*}{$\begin{array}{l}\text { Sinka, K; et al. } \\
2014\end{array}$} & \multirow[t]{3}{*}{86769} & \multirow{3}{*}{$\begin{array}{l}\text { UK. The Child Health System } \\
\text { database and the Scottish } \\
\text { Immunisation Recall System }\end{array}$} & \multirow[t]{3}{*}{ 2008-11 } & \multirow[t]{3}{*}{ HPV } & \multirow[t]{3}{*}{ 12-13 (catch up for 13-17) } & \multirow{3}{*}{$\begin{array}{l}\text { School-based (catch } \\
\text { up included supply } \\
\text { of vaccine at } \\
\text { primary health care) }\end{array}$} & Year 1: $89.4 \%$ & \multirow{3}{*}{$\begin{array}{l}\text { Scottish Index of Multiple } \\
\text { Deprivation (SIMD) }\end{array}$} \\
\hline & & & & & & & Year 2: $86.9 \%$ & \\
\hline & & & & & & & Year 3: $81 \%$ & \\
\hline $\begin{array}{l}\text { Markovitz, A; et } \\
\text { al. } 2014\end{array}$ & 13,709 & $\begin{array}{l}\text { USA. Immunization registry, } \\
\text { Michigan residents } \\
\text { continuously enrolled } \\
\text { with a PPO }\end{array}$ & 2006-11 & HPV & 11-12 (catch up to 26) & $\begin{array}{l}\text { Available at routine } \\
\text { healthcare provider }\end{array}$ & $22 \%$ & $\begin{array}{l}\text { Maternal preventive care } \\
\text { utilization (Pap testing, } \\
\text { mammograms, primary } \\
\text { care office visits), age, race, } \\
\text { household education, } \\
\text { household income, maternal } \\
\text { age. }\end{array}$ \\
\hline $\begin{array}{l}\text { Middleman, } \\
\text { A. } 2004\end{array}$ & 11,500 & USA. School data & $1998-2000$ & HBV & School grades 5-6 & School-based & $72 \%$ & Insurance, race and gender \\
\hline $\begin{array}{l}\text { Middleman, } \\
\text { A B; et al. } 1996\end{array}$ & 826 & $\begin{array}{l}\text { USA. Medical records from } \\
\text { an adolescent health clinic }\end{array}$ & Unknown & HBV & $\begin{array}{l}\text { Any adolescent } \\
\text { attending the } \\
\text { clinic }\end{array}$ & $\begin{array}{l}\text { Available at routine } \\
\text { healthcare provider }\end{array}$ & $23 \%$ & $\begin{array}{l}\text { Socio-demographics, risk } \\
\text { behaviors (for hepatitis B), } \\
\text { medication use, chronic } \\
\text { illnesses, and experience, } \\
\text { knowledge and attitudes } \\
\text { about hepatitis B and the } \\
\text { immunization }\end{array}$ \\
\hline $\begin{array}{l}\text { Middleman, } \\
\text { A B; et al. } 1999\end{array}$ & 943 & $\begin{array}{l}\text { USA. Questionnaires } \\
\text { distributed at hospital } \\
\text { and school based clinics }\end{array}$ & $1994-5$ & HBV & $\begin{array}{l}\text { Any adolescent } \\
\text { attending the } \\
\text { clinics }\end{array}$ & $\begin{array}{l}\text { Available at routine } \\
\text { healthcare provider } \\
\text { and school based } \\
\text { clinic }\end{array}$ & $\begin{array}{l}47.6 \% \text { (Clinic); } 41.7 \% \\
\text { (School-based clinic) }\end{array}$ & $\begin{array}{l}\text { Race, insurance, residential } \\
\text { zip code, risk factors for } \\
\text { acquiring hepatitis B, risk } \\
\text { behaviors (cigarette and } \\
\text { substance use), and academic } \\
\text { achievement, chronic illness, } \\
\text { healthcare utilization, } \\
\text { knowledge about hepatitis } \\
\text { B and the vaccination, family } \\
\text { history of hepatitis B } \\
\text { vaccination, travel time, } \\
\text { and mode of transportation } \\
\text { to the clinic. }\end{array}$ \\
\hline $\begin{array}{l}\text { Monnat, S; } \\
\text { Wallington, S. } \\
2013\end{array}$ & 4,776 & $\begin{array}{l}\text { USA. Behavioral Risk Factor } \\
\text { Surveillance System Survey } \\
\text { data in } 10 \text { territories }\end{array}$ & $2008-10$ & HPV & $11-12$ (catch up to 26) & $\begin{array}{l}\text { Available at routine } \\
\text { healthcare provider }\end{array}$ & $14 \%$ & $\begin{array}{l}\text { Mother's history of cervical } \\
\text { screening (Pap test). }\end{array}$ \\
\hline $\begin{array}{l}\text { MooreCaldwell, } \\
\text { S; et al. } 1997\end{array}$ & 174 & $\begin{array}{l}\text { USA. Medical records from } \\
\text { a university adolescent } \\
\text { clinic and junior-senior } \\
\text { private high school clinic }\end{array}$ & $1992-3$ & HBV & $\begin{array}{l}\text { Any adolescent } \\
\text { attending the } \\
\text { clinics }\end{array}$ & $\begin{array}{l}\text { Available at routine } \\
\text { healthcare provider } \\
\text { at school based } \\
\text { clinics }\end{array}$ & $89 \%$ & $\begin{array}{l}\text { Adolescent and parent } \\
\text { knowledge of hepatitis B, } \\
\text { perceived risk. }\end{array}$ \\
\hline
\end{tabular}


Table 3 Summary of included studies. Caption: a summary of the studies included in the review, including details of sample size, the source of the sample, year of data collection, vaccine investigated, target age group, delivery strategy, completion rate attained and factors investigated to influence completion (Continued)

\begin{tabular}{|c|c|c|c|c|c|c|c|c|}
\hline $\begin{array}{l}\text { Moss, J Li } \\
\text { et al. } 2013\end{array}$ & 105,121 & $\begin{array}{l}\text { USA. North Carolina } \\
\text { Immunization Registry }\end{array}$ & $\begin{array}{l}\text { Not } \\
\text { available }\end{array}$ & HPV & 11-12 (catch up to 26) & $\begin{array}{l}\text { Available at routine } \\
\text { healthcare provider }\end{array}$ & $28 \%$ & $\begin{array}{l}\text { Gender ratio, race, provider } \\
\text { specialty and adolescent } \\
\text { patient load, reminder/ } \\
\text { recall system, time to } \\
\text { documentation in NCIR, } \\
\text { computers per clinic, age } \\
\text { of vaccine recommendation } \\
\text { (Tdap, Meningococcal, HPV) }\end{array}$ \\
\hline \multirow{2}{*}{$\begin{array}{l}\text { Musto, R; } \\
\text { et al. } 2013\end{array}$} & \multirow[t]{2}{*}{35,592} & \multirow{2}{*}{$\begin{array}{l}\text { Canada. Calgary zone } \\
\text { Public Health vaccination } \\
\text { database }\end{array}$} & \multirow[t]{2}{*}{ 2008-11 } & \multirow[t]{2}{*}{ HPV } & \multirow[t]{2}{*}{ Grades 5 and 9} & \multirow{2}{*}{$\begin{array}{l}\text { School-based } \\
\text { programme and } \\
\text { available at } \\
\text { community public } \\
\text { health clinics }\end{array}$} & 75 \% (School-based); & \multirow{2}{*}{$\begin{array}{l}\text { In-school vs community } \\
\text { health clinic delivery model, } \\
\text { socioeconomic status, school } \\
\text { provider type, history of HBV. }\end{array}$} \\
\hline & & & & & & & 36 \% (community) & \\
\hline $\begin{array}{l}\text { Neubrand, T; } \\
\text { et al. } 2009\end{array}$ & 352 & $\begin{array}{l}\text { USA. Medical records } \\
\text { review from two different } \\
\text { sites }\end{array}$ & $2007-8$ & HPV & 11-12 (catch up to 26) & $\begin{array}{l}\text { Available at routine } \\
\text { healthcare provider }\end{array}$ & $58 \%$ & $\begin{array}{l}\text { Age, race, insurance (private } \\
\text { vs Medicaid/Child Health } \\
\text { Insurance Program [CHIP]), } \\
\text { and distance from home to } \\
\text { the clinic, sexual activity prior } \\
\text { to initiation of the series, } \\
\text { history of an STI, cervical } \\
\text { screening history within } 3 \\
\text { years of vaccine initiation, } \\
\text { reason for clinic visit }\end{array}$ \\
\hline $\begin{array}{l}\text { Niccolai, L; } \\
\text { et al. } 2011\end{array}$ & 7606 & $\begin{array}{l}\text { USA. NIS-Teen survey: } \\
\text { Random digit dialing } \\
\text { household survey. }\end{array}$ & 2008-9 & HPV & 11-12 (catch up to 26) & $\begin{array}{l}\text { Available at routine } \\
\text { healthcare provider }\end{array}$ & $55 \%$ & $\begin{array}{l}\text { Race, socioeconomic status, } \\
\text { age, maternal characteristics, } \\
\text { insurance, healthcare } \\
\text { utilization, geography } \\
\text { (region) and year. }\end{array}$ \\
\hline $\begin{array}{l}\text { Perkins, R B; } \\
\text { et al. } 2012\end{array}$ & 7702 & $\begin{array}{l}\text { USA. Electronic medical } \\
\text { records from Boston } \\
\text { Medical/ community } \\
\text { health centers }\end{array}$ & $2007-8$ & HPV & 11-12 (catch up to 26) & $\begin{array}{l}\text { Available at routine } \\
\text { healthcare provider }\end{array}$ & $20 \%$ & $\begin{array}{l}\text { Age, location, number of } \\
\text { clinic visits in study period, } \\
\text { race, risk behaviour } \\
\text { (documentation of STI or } \\
\text { alcohol use), history of } \\
\text { meningococcal or tdap } \\
\text { booster vaccine. }\end{array}$ \\
\hline $\begin{array}{l}\text { Pruitt, C N; } \\
\text { et al. } 2013\end{array}$ & 978 & $\begin{array}{l}\text { USA. Rochester Epidemiology } \\
\text { Project records (REP) from } \\
\text { medical records }\end{array}$ & 2006-9 & HPV & 11-12 (catch up to 26) & $\begin{array}{l}\text { Available at routine } \\
\text { healthcare provider }\end{array}$ & $\begin{array}{l}\text { Somali girls: } 52 \% \text {; } \\
\text { white/non-Hispanic: } \\
72 \%\end{array}$ & Somali ethnicity \\
\hline $\begin{array}{l}\text { Rahman, } \\
\text { et al. } 2013\end{array}$ & 2632 & $\begin{array}{l}\text { USA. Data from Behavioral } \\
\text { Risk Factor Surveillance } \\
\text { System Telephone survey }\end{array}$ & 2008-10 & HPV & 11-12 (catch up to 26) & $\begin{array}{l}\text { Available at routine } \\
\text { healthcare provider }\end{array}$ & $17 \%$ & Geography/ location \\
\hline $\begin{array}{l}\text { Reiter, P L; } \\
\text { et al. } 2009\end{array}$ & 229 & $\begin{array}{l}\text { USA. Telephone survey, } \\
\text { North Carolina. }\end{array}$ & $2007-8$ & HPV & 11-12 (catch up to 26) & $\begin{array}{l}\text { Available at routine } \\
\text { healthcare provider }\end{array}$ & $83 \%$ & $\begin{array}{l}\text { Adverse events/ reported } \\
\text { pain from HPV vaccination }\end{array}$ \\
\hline
\end{tabular}


Table 3 Summary of included studies. Caption: a summary of the studies included in the review, including details of sample size, the source of the sample, year of data collection, vaccine investigated, target age group, delivery strategy, completion rate attained and factors investigated to influence completion (Continued)

\begin{tabular}{|c|c|c|c|c|c|c|c|c|}
\hline $\begin{array}{l}\text { Reiter, } \mathrm{P} ; \\
\text { et al. } 2013\end{array}$ & 1951 & $\begin{array}{l}\text { USA. NIS-Teen survey: } \\
\text { Random digit telephone } \\
\text { survey }\end{array}$ & $2008-10$ & HPV & 11-12 (catch up to 26) & $\begin{array}{l}\text { Available at routine } \\
\text { healthcare provider }\end{array}$ & $28 \%$ & $\begin{array}{l}\text { Age, race, healthcare utilization } \\
\text { in last year, insurance, } \\
\text { maternal characteristics, } \\
\text { knowledge of HPV, provider } \\
\text { recommendation, } \\
\text { socioeconomic status. }\end{array}$ \\
\hline $\begin{array}{l}\text { Rouzier, R; } \\
\text { Giordanella, } \\
\text { J. } 2010\end{array}$ & 77,744 & $\begin{array}{l}\text { France. CPAM social } \\
\text { security database }\end{array}$ & $2007-8$ & HPV & 14 (Catch up to 23) & $\begin{array}{l}\text { Available at routine } \\
\text { healthcare provider }\end{array}$ & $43 \%$ & $\begin{array}{l}\text { Age, provider (general } \\
\text { practitioner vs. gynecologist) }\end{array}$ \\
\hline $\begin{array}{l}\text { Rubin, R; } \\
\text { et al. } 2012\end{array}$ & 10,821 & $\begin{array}{l}\text { USA, Administrative } \\
\text { reimbursement data and } \\
\text { medical records from } \\
\text { medical group practices }\end{array}$ & $2006-10$ & HPV & $11-12$ (catch up to 26) & $\begin{array}{l}\text { Available at routine } \\
\text { healthcare provider }\end{array}$ & $27 \%$ & $\begin{array}{l}\text { Pre-existing STD, age, provider } \\
\text { medical department }\end{array}$ \\
\hline $\begin{array}{l}\text { Sakou, I l; } \\
\text { et al. } 2011\end{array}$ & 1005 & $\begin{array}{l}\text { Greece. Convenience sample } \\
\text { of Adolescent Health Unit } \\
\text { attendees }\end{array}$ & 2009 & $\begin{array}{l}\text { HBV, } \\
\text { HAV, } \\
\text { HPV }\end{array}$ & $\begin{array}{l}\text { HPV: } 12-15 ; \text { HAV, } \\
\text { HBV: catch up 11-18 }\end{array}$ & $\begin{array}{l}\text { Available at routine } \\
\text { healthcare provider }\end{array}$ & Not reported & $\begin{array}{l}\text { Gender, race/ nationality, } \\
\text { parental education, family } \\
\text { status }\end{array}$ \\
\hline $\begin{array}{l}\text { Schluterman, } \\
\text { N H; et al. } 2011\end{array}$ & 8069 & $\begin{array}{l}\text { USA. Database of the } \\
\text { University of Maryland } \\
\text { Medical Center (UMMC) }\end{array}$ & $2006-10$ & HPV & 11-12 (catch up to 26) & $\begin{array}{l}\text { Available at routine } \\
\text { healthcare provider }\end{array}$ & $11 \%$ & $\begin{array}{l}\text { Race, insurance status } \\
\text { (publicly funded, private, } \\
\text { or none), age (9-13, 14-17, } \\
\text { or } 18-26 \text { years), and place } \\
\text { of residence (urban or } \\
\text { suburban Baltimore). }\end{array}$ \\
\hline $\begin{array}{l}\text { Schmidt, M } \\
\text { A; et al. } 2013\end{array}$ & 311213 & $\begin{array}{l}\text { USA. Administrative data } \\
\text { from vaccination sites }\end{array}$ & 2006-11 & HPV & 11-12 (catch up to 26) & $\begin{array}{l}\text { Available at routine } \\
\text { healthcare provider }\end{array}$ & $42 \%$ & Age, calendar year \\
\hline $\begin{array}{l}\text { Schmitt, K; } \\
\text { Thompson, } \\
\text { D. } 2013\end{array}$ & $\mathrm{n} / \mathrm{a}$ & $\begin{array}{l}\text { USA. Statewide } \\
\text { Immunization Registry }\end{array}$ & $2001-11$ & HPV & $11-12$ (catch up to 26) & $\begin{array}{l}\text { Available at routine } \\
\text { healthcare provider }\end{array}$ & $52 \%$ & $\begin{array}{l}\text { Age, insurance, provider } \\
\text { type, race }\end{array}$ \\
\hline $\begin{array}{l}\text { Seid, M; } \\
\text { et al. } 2001\end{array}$ & 800 & $\begin{array}{l}\text { USA. Survey to parents of } \\
\text { children at } 5 \text { Schools, } \\
\text { San Diego }\end{array}$ & 1998 & HBV & $11-12$ & $\begin{array}{l}\text { Available at routine } \\
\text { healthcare provider }\end{array}$ & $27 \%$ & $\begin{array}{l}\text { Provider, school based } \\
\text { clinics, school socioeconomic } \\
\text { status, home language, race, } \\
\text { insurance, health care } \\
\text { utilization, heard about } \\
\text { mandatory vaccination } \\
\text { from health care provider. }\end{array}$ \\
\hline $\begin{array}{l}\text { Smith, L M; } \\
\text { et al. } 2011\end{array}$ & 2519 & $\begin{array}{l}\text { Canada. Universal health } \\
\text { insurance program database. }\end{array}$ & $2007-10$ & HPV & School grade 8 & School-based & $86 \%$ & $\begin{array}{l}\text { Age, parental income, and } \\
\text { place of residence, vaccination } \\
\text { history, health services } \\
\text { utilisation, medical history. }\end{array}$ \\
\hline $\begin{array}{l}\text { Tan, W; } \\
\text { et al. } 2011\end{array}$ & 138823 & $\begin{array}{l}\text { USA. NCIR immunisation } \\
\text { registry }\end{array}$ & $2006-2009$ & HPV & 11-12 (catch up to 26) & $\begin{array}{l}\text { Available at routine } \\
\text { healthcare provider }\end{array}$ & $55 \%$ & $\begin{array}{l}\text { Race, age, county of residence, } \\
\text { provider clinic type, insurance. }\end{array}$ \\
\hline
\end{tabular}


Table 3 Summary of included studies. Caption: a summary of the studies included in the review, including details of sample size, the source of the sample, year of data collection, vaccine investigated, target age group, delivery strategy, completion rate attained and factors investigated to influence completion (Continued)

\begin{tabular}{|c|c|c|c|c|c|c|c|c|}
\hline \multirow{2}{*}{$\begin{array}{l}\text { Teplow-Phipps, } \\
\text { R; et al. } 2014\end{array}$} & \multirow[t]{2}{*}{1,494767} & \multirow{2}{*}{$\begin{array}{l}\text { USA. Citywide Immunization } \\
\text { Registry (CIR), New York City }\end{array}$} & \multirow[t]{2}{*}{$2005-12$} & \multirow[t]{2}{*}{ HPV } & \multirow[t]{2}{*}{$11-12$ (catch up to 26$)$} & \multirow{2}{*}{$\begin{array}{l}\text { Available at routine } \\
\text { healthcare provider }\end{array}$} & $38.4 \%$ (females) & \multirow{2}{*}{$\begin{array}{l}\text { Age, gender, insurance, clinic } \\
\text { specific variables: provider } \\
\text { practice-type, number of } \\
\text { Tdap vaccines reported } \\
\text { (proxy for practice size), } \\
\text { and socioeconomic status } \\
\text { of practice location. }\end{array}$} \\
\hline & & & & & & & 35.7 \% (males) & \\
\hline $\begin{array}{l}\text { Tracy, J K; } \\
\text { et al. } 2010\end{array}$ & 9658 & $\begin{array}{l}\text { USA. Clinical data repository } \\
\text { at the University of Maryland } \\
\text { Medical Center }\end{array}$ & 2006-10 & HPV & 11-12 (catch up to 26) & $\begin{array}{l}\text { Available at routine } \\
\text { healthcare provider }\end{array}$ & $31 \%$ & Age, race. \\
\hline $\begin{array}{l}\text { Tung, C S; } \\
\text { Middleman, } \\
\text { A B. } 2005\end{array}$ & 8918 & $\begin{array}{l}\text { USA, Data from } 75 \text { schools } \\
\text { participating in HBll (Hep } \\
\text { B immunization initiative). }\end{array}$ & $1999-2000$ & HBV & $13-15$ & School-based & $59 \%$ & $\begin{array}{l}\text { Publicity/promotion, packet } \\
\text { distribution, return of forms, } \\
\text { ratio of students to clinic, } \\
\text { provider characteristics }\end{array}$ \\
\hline $\begin{array}{l}\text { Verdenius, l; } \\
\text { et al. } 2013\end{array}$ & 1563 & $\begin{array}{l}\text { USA. Electronic medical } \\
\text { records }\end{array}$ & $2006-9$ & HPV & 11-12 (catch up to 26) & $\begin{array}{l}\text { Available at routine } \\
\text { healthcare provider }\end{array}$ & $32 \%$ & $\begin{array}{l}\text { Age, type of health visit, } \\
\text { healthcare utilization, } \\
\text { concomitant healthcare } \\
\text { delivery. }\end{array}$ \\
\hline $\begin{array}{l}\text { Widdice, L E; } \\
\text { et al. } 2011\end{array}$ & 3297 & $\begin{array}{l}\text { USA. Review of medical } \\
\text { records from academic } \\
\text { medical center }\end{array}$ & $2006-8$ & HPV & 11-12 (catch up to 26) & $\begin{array}{l}\text { Available at routine } \\
\text { healthcare provider }\end{array}$ & $28 \%$ & $\begin{array}{l}\text { Age, race, insurance, } \\
\text { healthcare utilization (DMPA), } \\
\text { clinic location, time period o } \\
\text { f vaccine series initiation }\end{array}$ \\
\hline $\begin{array}{l}\text { LaMontagne, } \\
\text { D; et al. } 2011\end{array}$ & 7269 & $\begin{array}{l}\text { Peru, India, Uganda, } \\
\text { Vietnam. Population based } \\
\text { household survey }\end{array}$ & 2008-10 & HPV & $\begin{array}{l}\text { Peru: grade } 5 \text {; Uganda: } \\
\text { grade } 5 \text { or age } 10 ; \\
\text { Vietnam: grade } 6 \text { or } \\
\text { age } 11 ; \text { India: } 10-14 \text {. }\end{array}$ & $\begin{array}{l}\text { School-based or } \\
\text { health centre based } \\
\text { in all } 4 \text { countries }\end{array}$ & Not reported & Delivery Strategy \\
\hline
\end{tabular}


Table 4 Data available on factors investigated across countries and vaccines

\begin{tabular}{llll}
\hline Factor investigated & $\begin{array}{l}\text { Countries (Number of studies } \\
\text { with multivariate analyses) }\end{array}$ & Vaccine & HBV \\
\cline { 2 - 4 } Age & USA (17), Canada (1), France (1), Australia (1) & $\checkmark$ & HAV \\
Race & USA (16), Australia (1), Greece (1) & $\checkmark$ & $\checkmark$ \\
Insurance & USA (15), France (1) & $\checkmark$ & $\checkmark$ \\
Gender & Australia (1), USA (2) & $\checkmark$ & $\checkmark$ \\
Socio-economic status & USA (11), Canada (1), UK (1), France (1) & $\checkmark$ & $\checkmark$ \\
Healthcare utilization & USA (14), France (1), Australia (1), Canada (1) & $\checkmark$ & $\checkmark$ \\
Vaccine knowledge & USA (3) & $\checkmark$ & $\checkmark$ \\
Adverse events & USA (3) & $\checkmark$ & $\checkmark$ \\
Risk behaviour & USA (3), Australia (1) & $\checkmark$ & $\checkmark$ \\
Concomitant healthcare & USA (3) & $\checkmark$ & $\checkmark$ \\
Access & USA (2) & $\checkmark$ & $\checkmark$ \\
Maternal characteristics & Pap smear history - USA (3) & $\checkmark$ & $\checkmark$ \\
& Education - USA (7), Greece (1) & & $\checkmark$ \\
\end{tabular}

(aOR 0.75; 0.72-0.79) women had 25-45 \% lower likelihood of completion compared to White women [43]. Race was the only significant predictor of completion in the NIS-Teen household survey in USA [44]. Ten additional large database studies in the USA with multivariate analyses corroborate this association for both HPV and $H B V$ vaccines [15, 16, 36, 37, 39, 40, 42, 43, 46-48]. However, no association between race and completion was found in 5 studies when controlling for gender, insurance and health clinic characteristics [29, 38, 45, 49, 50]. Hispanic adolescents were underrepresented in one survey with a low response rate [29].

Greek non-nationals had lower completion rates (33\%) than nationals (60\%) for 2 doses of HAV [51]. In the northern territories of Australia, 3 dose coverage of $\mathrm{HPV}$ vaccine was lower in indigenous compared to nonindigenous groups (54\% vs. $64 \%$ ), but completion rates were the same (84\%) [52].

\section{Insurance}

Many countries have supplied HPV vaccine free-ofcharge. In the USA, although the vaccine was not initially eligible for reimbursement in some health insurance plans, after it was recommended by the Advisory Committee on Immunization Practices it was included in the Vaccines for Children (VFC) programme which provides for underinsured and uninsured children [1]. Insurance status was investigated as a risk factor in 25 articles, 16 conducted multivariate analyses (15 USA, 1 France). In 2011, insurance status remained a significant predictor of HPV series completion in the USA; those publicly insured (Medicaid) were 2.08 times (95 \% CI 1.16-3.7) more likely to complete compared to those with no insurance; those privately insured were not significantly more likely to complete than those on public insurance (aOR 1.16; 95 \% CI 0.97-1.38) controlling for age, race, contraception use [42]. The association between insurance status and completion was stronger in 2006-8 reflecting policy changes [16, 43]. In France completion rates were lower among recipients of complimentary social welfare compared to those with private insurance (aRR 0.88; $95 \%$ CI 0.83-0.93) [34].

Longer enrolment on an insurance plan ( $>12$ years) was associated with a 1-14\% increase in likelihood of completion of 2 doses of varicella vaccine; $9-12 \%$ increased likelihood for HAV and 21-23\% for HBV in the vaccine safety database of almost 600,000 people in the USA between 1998 and 2004 [31] controlling for age, gender, healthcare utilisation and provider characteristics.

Across the USA there are substantial differences across states in beliefs, policy, and the rapidity of implementation of changes made at the national level. In Oregon state in 2008, HPV vaccine was offered free of charge and no difference was found in completion rates between publicly and privately insured participants [53]. In Maryland in 2006-10 private insurance was found to be a risk factor for noncompletion compared to those publicly insured (aOR 0.76; $95 \%$ CI 0.59-0.98), controlling for race and age [37]. No association in multivariate analyses was seen in 5 studies in the USA $[29,39,40,44,50]$.

\section{Gender}

Gender was assessed in seven articles; no correlation between completion of HBV and gender was seen in unadjusted results from Greece [51], nor in adjusted results in Australia [35]. In the USA, controlling for delivery site, age, insurance, year, chronic conditions and 
prior healthcare utilization, male gender was marginally associated with lower completion for varicella (aOR 0.93; 95 \% CI 0.90-0.96), HAV (aOR 0.98; 0.97-0.99) and HBV (aOR 0.97; 0.96-0.98) [31]. Included studies did not report completion of HPV vaccine in boys, recommendations to vaccinate boys were issued in 2015 in the USA; however, clinics in the USA with higher female:male ratios obtained higher completion rates of HPV vaccine among females (aRR 2.16; 1.13-4.13) [49].

\section{Socio-economic status}

Socio-economic status (SES) was analysed in studies in the USA $(n=14)$, Canada $(n=2)$, UK $(n=1)$ and France $(n=1) ; 14$ conducted multivariate analysis. Median neighbourhood income and average adult education [54], parental income levels [39, 50, 55], household income [56] and poverty status [45] were not associated with completion in multivariate analyses.

Every 10,000USD rise in median neighbourhood income was associated with a $15 \%$ increase in HBV completion (aRR 1.15; $95 \%$ CI 1.06-1.25) [47] and a $1 \%$ increased likelihood of HPV completion in 20,000 9-17 year old American girls (aRR 1.01; 1.01-1.02) [57]. Average census block education level was positively associated at a similar magnitude of effect (aRR 1.03; 1.02-1.05) [57]. Adolescent girls living below the federal poverty level were significantly less likely to complete vaccination compared to adolescents with household incomes $>\$ 75,000$ (aOR 0.76; 0.63-0.92) [44].

The effect of SES may differ by delivery strategy; in Canadian public schools with in-school HPV vaccine delivery, completion increased as SES decreased, in Catholic schools in which the pupils relied on community delivery, completion decreased as SES decreased [58]. A linear trend with the Scottish multiple index of deprivation was found with completion but not with initiation; however, the difference between the most and least deprived groups was small (8\%) and disappeared with the administration of a catch-up dose 1 year later [59]. Girls in Canada in 2007-8 living in lower income neighbourhoods were significantly less likely to complete HPV vaccine than girls living in middle income neighbourhoods (aOR 0.45; 0.28-0.72) [33]. In France compliance with the HPV vaccine schedule was lower in social welfare recipients compared to nonrecipients (aRR 0.88, 0.83-0.93) [34].

\section{Healthcare utilization}

History of health care utilization was inconsistently associated with completion. Seventeen articles from the USA, France and Australia analysed an individual's prior use of health care (defined by receipt of other recommended vaccines, or the number of prior visits to a primary health care provider) and completion of a multi-dose series of varicella, HPV or HBV vaccines. In adjusted analyses in the USA, $>10$ visits to a health care provider in the last year was associated with $15 \%$ increased likelihood of HPV vaccine completion and a 4-6 \% increase in HBV completion [31]. Similar findings were reported in France where compliance with the HPV vaccine regimen was $10 \%$ higher if a girl had $>6$ consultations with a family physician in the past year [34]. The magnitude of the effect is supported by reports of a $2 \%$ increased likelihood of completing the HPV vaccine series with every primary care provider visit in the past year [36].

A further eight studies found no association between vaccine completion and the number of visits to a primary healthcare in the preceding 2 years [60], non-acute care in the year preceding initiation $[29,33,39,44,55]$, previous prescriptions [47] or receipt of tetanus, diphtheria, and pertussis booster (Tdap) and meningococcal vaccines [38].

Recorded contraceptive use (DMPA) at any time in the medical records by HPV vaccine recipients was associated with a two-fold increase (95\% CI 1.72-2.47) in the odds of HPV vaccine completion [42]. In Canada, HBV vaccination conferred 16.9 times higher odds $(95 \%$ CI 14.8-19.2) for HPV vaccine completion in comparison with those who had not received HBV. However, the association could be confounded by the differing vaccination policies and delivery strategies by school [58]. In Australia in an area with a high risk population, including young sex workers and drug users, a shorter time interval ( $<2$ weeks) between first contact with the health care provider and initiation of vaccine series correlated with better HBV completion [35].

\section{Vaccine related knowledge}

Three American studies examined knowledge in relation to completion in multivariate analysis $[45,47,55]$. The ability to correctly identify the number of required doses remained associated with series completion (aRR 1.38; 95 \% CI 1.08-1.76) [55]. Parents who remember receiving a provider recommendation for vaccination were more likely to have daughters who had completed the regimen (aOR 2.71; 1.99-3.70) [45]. However, general knowledge of HPV and HBV vaccine was not associated with completion in adjusted analyses [45, 47].

\section{Adverse events}

Three studies assessed whether experience of adverse events following HPV vaccination affected series completion in the USA. Parents of daughters who had completed the three dose series reported pain or discomfort as often as parents whose daughters were late for their second or third dose (OR 0.76; $95 \%$ CI 0.33-1.77) [61]. In a survey of over 3000 vaccine recipients [55] 
(response rate $27 \%$ ), multivariate analysis controlling for age, socio-economic status, health care utilization, showed reports of bruising or swelling at first dose did not affect completion of the series (aRR 0.88; 0.7-1.00). An association was not apparent for those reporting pain, syncope or dizziness [55]. A qualitative study of 18 women in the USA who did not complete the HPV vaccine series found none of them mentioned adverse events as a reason [62].

\section{Risk behaviour}

A variety of risk behaviours in seven studies were assessed in relation to completion of HBV or HPV vaccine schedules; no associations were found. Drug use, history of sexually transmitted infections (STIs), or alcohol use was not associated with completion in the USA $[38,47,63]$. In multivariate analysis in Australia, intravenous drug use, sex work, or hepatitis $C$ status did not correlate with likelihood of completion of HPV in a health unit serving at-risk populations [35].

\section{Concomittant healthcare}

Three articles assessed the effect of concomitant health service delivery on adherence to HPV in the USA. Receipt of another vaccine at the time of HPV vaccination was not associated with odds of HPV completion controlling for socio-demographic and provider characteristics [55]. However, if the first dose was given at a health care provider visit which was attended primarily for another reason other than $\mathrm{HPV}$, the odds of a mistimed 3rd dose were almost double (aOR 1.97; $95 \%$ CI 1.39-2.80) than that if the first dose was at a vaccine only visit, controlling for age and race [48]. Type of visit was not associated in analysis investigating the effect of age and healthcare utilization [41].

\section{Access}

Access to vaccination sites was assessed in two studies in the USA. Compliance to the schedule and completion of the series were not governed by proximity or mode of transportation to the clinical site [47]. Distance from home to clinic was not associated with completion controlling for age, race, and healthcare utilization [40].

\section{Qualitative studies}

One qualitative study investigated why 9-26 year olds did not return for the final dose of the HPV vaccine series, in non-exclusive responses: $33 \%$ claimed they didn't know they were meant to obtain further doses, $23 \%$ claimed they were too busy, $15 \%$ cited inconvenience, $38.5 \%$ claimed they were too busy or forgot, $7.7 \%$ claimed they were too busy and times were inconvenient
[62]. Two additional surveys of partially vaccinated university students in the USA and Australia indicated the potential problems with vaccinating older age groups who have competing priorities; reasons focused on inconvenience and lack of time $[64,65]$.

\section{Maternal characteristics}

Three studies in the USA analyzed the relationship between maternal preventative behavior (cervical screening) and their daughter's HPV vaccine series completion. In multivariate analysis, controlling for demographic, socioeconomic, family, and health plan characteristics, all three studies found that girls whose mothers had received a pap smear in the past three years were more likely to complete the HPV vaccine series (aOR 1.07, $95 \%$ CI 1.06-1.08) [56]; 1.42, 1.31-1.54 [54] and 1.87, 1.31-2.75 [66]).

The relationship between maternal education and vaccine series completion was assessed in eight studies conducted in the USA $(n=7)$ and in Greece [51]. Adolescents whose mothers had less than high school education were less likely to complete the vaccine series in multivariate analysis [44, 66]; both studies controlled for adolescent age, SES, and mother's health characteristics and found similar effect estimates (aOR 0.68; $95 \%$ CI 0.56-0.84) [44]; aOR 0.60; 0.41-0.87 [66]). No association between maternal education and HPV or HAV vaccine series completion in multivariate analysis was found in three studies $[39,45,50]$.

Maternal age and marital status were found to have no or very slight associations with vaccine series completion in four of the five included studies $[39,44,45,66]$. In unadjusted analysis, one study found daughters with mothers aged over 40 years were more likely to complete the HPV vaccine series compared to mothers who were less than 40 years old [56].

\section{Provider/ organisational characteristics Delivery model}

There is strong evidence for high completion rates with school-delivery in high - income and low-middle income countries. Canadian in-school HPV vaccination completion rates were $75 \%$ (95\% CI 74.7-75.8) compared to $36 \%$ (95 \% CI 35.3-37.2) for girls provided with a community-delivery model [58]. In-school vaccinations conferred 1.8 times the odds of completing the HBV series compared to if adolescents had to go off-site (95\% CI 1.15-2.8) in a parent survey in the USA controlling for age, race, insurance, SES, prior healthcare utilization [29].

Only 2 articles included data from low and middle income countries (LMIC); descriptive results are available regarding the success of different delivery strategies [67]. In Uganda, a school-based strategy appeared more successful (94\% completion) than a strategy in which the vaccine was given in the community with a child 
health programme (79-87\% completion year 1-year 2) although the delivery strategies had slightly different target populations. Peru's school-based strategy achieved $98.7 \%$ completion, whilst combined school-based and health centre strategies in Vietnam achieved $>99 \%$ completion. In India, very similar completion rates were achieved in campaign and routine delivery approaches (97-98 \%) [67]. Differences in completion rates achieved in 21 demonstration projects in 14 countries implementing different models of delivery were insignificant [68]; however, the mixed model (school based delivery with mop-up activities at health centres) seemed to confer marginally higher completion (96.6\%), the school-only model was intermediate $(88.6 \%)$ and the health facilityonly model was least effective $(79.7 \%)(p=0.39)$ [69].

In Australia, high-risk groups, benefited from an accelerated schedule $(0,7,21$ days and 12 months), which increased the likelihood of HBV vaccine completion 1.35 times (1.01-1.80) controlling for drug use, and length of contact with the health facility [35].

\section{Provider characteristics}

Vaccine schedule completion was higher in an American school based programme when students returned the consent forms to their teacher compared to the school nurse [70]. In 1994-5 in Canada, initial parental consent was lower at private schools compared to public schools; however, private and public schools did not differ in completion rates. Different education providers (teachers or public health nurses) did not have an effect on completion, although education from teachers was associated with higher consent [30].

A further 17 studies investigated health provider characteristics, of which 12 reported adjusted analyses. There was no evidence that the speciality of an adolescent girl's primary care physician influenced HPV series completion in multivariate analyses [15, 16, 36, 42, 49, 55]. However, for women $>17$ years of age in the USA, those with a paediatric/internal medicine physician were slightly less likely to complete the HPV regimen than those with a family medicine physician. Female providers were not significantly associated with completion (male primary care provider aRR0.93 0.85-1.01) [36]. In an American datalink study, those vaccinated at paediatric clinics had the highest completion (61\%) compared to family care practices (53 \%; aOR 0.78; $95 \%$ CI 0.76-0.80) and the local health departments (39\%; aOR 0.48; 0.47-0.50) [43].

\section{Discussion}

We present a comprehensive review of the available literature on factors influencing adherence to multi-dose vaccine schedules among adolescents. The majority of studies took place in the USA $(n=49)$, the remainder included Canada $(n=3)$, France $(n=3)$, Australia $(n=2)$,
Greece $(n=1)$, the UK $(n=1)$ and 2 multi-country studies including LMICs. The two studies including LMICs focused on organisational level factors and reported high adherence to HPV vaccine [68], therefore our summaries of individual level factors are limited in generalizability to developed settings. The high level of variation in the definitions, number and selection of factors investigated in each study limits the comparability of study results and prevented conduct of a meta-analysis. The overall impact of the identified characteristics on vaccine adherence is likely to be dependent on the mix of other factors present, as well as the programmatic and local context.

Good adherence to multi-dose vaccines appears to be higher in early adolescence (9-12 year olds) when compared to later adolescence ( $>14$ years old). It is unclear whether this is linked to adolescent health seeking behaviour, which was inconsistently associated with completion, or whether it is governed by logistical reasons as cited in qualitative results. It could reflect factors which are not explored in the available literature such as which groups were most targeted with communication materials or the general decrease in utilization of health services through adolescence [71]. In some populations in the USA, there is evidence that Black or Hispanic girls are disproportionately prone to low completion rates when compared to White girls after adjustment for socioeconomic status and insurance, despite some reports of similar rates of initiation. Adolescent females may have a slightly elevated likelihood of vaccine completion compared to males; this association may be a symptom of increased opportunity whilst accessing contraception at the health centre. Higher household income, maternal education and maternal preventative health behaviour were associated with higher completion rates when compared to lower socio-economic families and those mothers who rarely sought screening. Insurance status may have a decreasing effect on completion over time as knowledge spreads that both HBV and HPV vaccines are eligible for reimbursement on any insurance plan in the USA. Experience of adverse events and general knowledge about the vaccine did not affect completion rates. School-based delivery alongside supplying vaccine to health centres for out-of-school girls appears to be a successful approach in countries with relatively high school attendance, including some LMICs [69], the UK [59] and Canada [58].

\section{Conclusions}

The factors which affect rates of vaccine completion are context and time specific. Providers and programme planners should be aware that obtaining good consent and initiation rates is not enough; sub-groups within the population may need more help than others to complete 
the series. Efforts need to continue past the first dose to reduce inequality in completion. Adolescents captured for the first dose remain only partially protected from vaccine related disease until receipt of the final dose of the schedule.

Opportunistic vaccination at the delivery point of other services should be utilized as a strategy to increase vaccine completion. There is no evidence that concomitant service delivery is associated with lower completion. Among 11-18 year olds in Seattle, $71 \%$ of visits to a primary health practitioner in 2006-11 were found to be lost opportunities for dose 3 of HPV vaccine [72]. Especially utilizing visits which were not originally for preventative health care services could rapidly improve completion rates and access those adolescents with low healthcare utilization $[71,72]$.

A Cochrane review in 2005 found 47 articles detailing the effect of patient reminder/recall on vaccine uptake, all were conducted in developed countries, only one study was conducted in adolescents [73]. In pooled results across all age groups, all reminder/recall systems appeared to improve coverage compared to the control groups. Personal telephone reminders were the most effective intervention (OR 1.92; 95 \% CI 1.2-3.07). Letter reminders were close to the effectiveness of phone reminders (OR 1.79; 1.5-2.15), a postcard alone was less effective (OR 1.44; 1.09-1.89), and automated phone calls were least effective (OR 1.29; 1.09-1.53). Interventions to improve completion of vaccine series need to be assessed and the use of novel technologies needs to be explored where electronic records and recall systems are not available.

\section{Additional file}

Additional file 1: Table S1. Search terms and results Medline (Ovid SP)1946 to 2014-02-26. (DOCX 17 kb)

\section{Competing interests}

The authors declare that they have no competing interests.

\section{Authors' contributions}

KEG carried out article screening, synthesis and drafted the manuscript. EK carried out article screening and synthesis. LOE carried out article screening. SM carried out article screening. SM-J carried out article screening (French). MA carried out article screening (Spanish). DAR provided input on the design and methods. DWJ provided input on the design and methods. All authors read, revised and approved the final manuscript.

\section{Acknowledgements}

The corresponding author is funded by a vaccine research fellowship from the Medical Research Council, UK. The work of the Catalan Institute of Oncology was partially funded by public grants from the Instituto de Salud Carlos III (grants references RD12/0036/0056 and CIBERESP), from the Agència de Gestió d'Ajuts Universitaris i de Recerca (grants references 2014SGR1077 and 2014SGR2016) and from the European Community's Seventh Framework Programme (grant agreement No.603019, Project CoheaHr). None of the sources of funding had any role in the preparation of this article.

\section{Author details}

'Clinical Research Department, Faculty of Infectious Tropical Diseases, London School of Hygiene and Tropical Medicine, Keppel Street, London WC1E 7HT, UK. ${ }^{2}$ Mwanza Intervention Trials Unit, The National Institute for Medical Research Mwanza Campus, PO Box 11936, Mwanza, Tanzania. ${ }^{3}$ Department of Epidemiology, University of Washington, 1959 NE Pacific Street, Health Sciences Building F-250, Box 357236, Seattle, WA 98195-7236, USA. ${ }^{4}$ Departments of Obstetrics and Gynaecology and Global Health, University of Washington, 1959 NE Pacific Street, Health Sciences Building F-250, Box 357236, Seattle, WA 98195-7236, USA. ${ }^{5}$ Department of Global Health and Development, Faculty of Public Health and Policy, London School of Hygiene and Tropical Medicine, 15-17 Tavistock Place, London WC1H 9SH, UK. Infections and Cancer Unit, Cancer Epidemiology Research Programme, Catalan Institute of Oncology, Av. Gran Via de I'Hospitalet 199-203, Hospitalet de Llobregat, 08908 Barcelona, Spain. ${ }^{7}$ Bellvitge Institute of Biomedical Research (IDIBELL), Barcelona, Spain. ${ }^{8} \mathrm{MRC}$ Tropical Epidemiology Group, Faculty of Epidemiology and Population Health, London school of Hygiene and Tropical Medicine, Keppel Street, London WC1E 7HT, UK.

Received: 10 September 2015 Accepted: 8 February 2016

Published online: 19 February 2016

\section{References}

1. Beharry MS, Coles MS, Burstein GR. Adolescent immunization update. Pediatr Infect Dis J. 2011;30(9):787-90.

2. Ackerman LK. Update on immunizations in children and adolescents. Am Fam Physician. 2008;77(11):1561-8.

3. GAVI Alliance. http://www.gavialliance.org/ [cited 30/07/2014]

4. World Health Organization. Global database of EPI Schedules. http://apps. who.int/immunization_monitoring/globalsummary/schedules. [cited 11 January 2015]

5. World Health Organization. Hepatitis B vaccine - WHO position paper in weekly epidemiological record. Geneva: WHO; 2009. p. 405

6. World Health Organization. WHO recommendations for routine immunization - summary tables - http://www.who.int/immunization/policy/ immunization_tables/en/. [cited 11 December 2014].

7. World Health Organization. Varicella vaccines, WHO position paper. In: Weekly epidemiological record. 1998. p. 241-8.

8. World Health Organization, World Health Organization, Human Papillomavirus vaccines. WHO position paper. Wkly Epidemiol Rec. 2009; 84(1):118-31.

9. World Health Organization. Human Papillomavirus vaccines: WHO position paper October 2014. In: Weekly epidemiological record. 2014. p. 465-92.

10. Strategic Advisory Group of Experts (SAGE) on Immunization. Evidence based recommendations on Human Papilloma Virus (HPV) vaccines schedules: background paper for SAGE discussions. Geneva: World Health Organization; 2014.

11. Lazcano-Ponce $E$ et al. Overcoming barriers to HPV vaccination: noninferiority of antibody response to human papillomavirus 16/18 vaccine in adolescents vaccinated with a two-dose vs. a three-dose schedule at 21 months. Vaccine. 2014;32(6):725-32.

12. Dobson SR et al. Immunogenicity of 2 doses of HPV vaccine in younger adolescents vs 3 doses in young women: a randomized clinical trial. JAMA. 2013;309(17):1793-802.

13. Baldo $V$ et al. Varicella: epidemiological aspects and vaccination coverage in the Veneto Region. BMC Infect Dis. 2009;9.

14. World Health Organization. WHO position paper on hepatitis A vaccines June 2012. In: Weekly epidemiological record. 2012. p. 261-76.

15. Cook RL et al. Factors associated with initiation and completion of human papillomavirus vaccine series among young women enrolled in Medicaid. J Adolesc Health. 2010;47(6):596-9.

16. Chou B et al. Disparities in human papillomavirus vaccine completion among vaccine initiators. Obstet Gynecol. 2011;118(1):14-20.

17. Kessels SJ et al. Factors associated with HPV vaccine uptake in teenage girls: a systematic review. Vaccine. 2012;30(24):3546-56.

18. Lehmann C, Benson PA. Vaccine adherence in adolescents. Clin Pediatr (Phila). 2009:48(8):801-11.

19. Falagas ME, Zarkadoulia E. Factors associated with suboptimal compliance to vaccinations in children in developed countries: a systematic review. Curr Med Res Opin. 2008;24(6):1719-41. 
20. Katz IT et al. Scaling up human papillomavirus vaccination: a conceptual framework of vaccine adherence. Sex Health. 2010;7(3):279-86.

21. Etter DJ, Zimet GD, Rickert VI. Human papillomavirus vaccine in adolescent women: a 2012 update. Curr Opin Obstet Gynecol. 2012;24(5):305-10.

22. Briss PA et al. Reviews of evidence regarding interventions to improve vaccination coverage in children, adolescents, and adults. Am J Prev Med. 2000;18(1, Supplement 1):97-140.

23. Cochrane Child Health Group. The cochrane child health field. Resources for producing child health reviews http://childhealth.cochrane.org/producingchild-relevant-cochrane-review. [cited 11 January 2014].

24. Centers for Disease, Control and Prevention. Recommended vaccine schedules http://www.cdc.gov/vaccines/default.htm. [cited 22 October 2015].

25. World Health Organization. WHO prequalified vaccines list. 1st April 2014. http://www.who.int/immunization_standards/vaccine_quality/PQ_vaccine_ list_en/en/. [cited 9 December 2014].

26. Moher $\mathrm{D}$ et al. Preferred reporting items for systematic reviews and metaanalyses: the PRISMA statement. Int J Surg. 2010;8(5):336-41.

27. Higgins J, Green S. Cochrane handbook for systematic reviews of interventions version 5.1.0 [updated March 2011] Available from www. cochrane-handbook.org. 2011, The Cochrane Collaboration.

28. Binagwaho A et al. Achieving high coverage in Rwanda's national human papillomavirus vaccination programme. Bull World Health Organ. 2012;90(8): 623-8.

29. Seid $\mathrm{M}$ et al. Correlates of vaccination for hepatitis B among adolescents. Results from a parent survey. Arch Pediatr Adolesc Med. 2001;155:921-6.

30. Deeks SL, Johnson IL. Vaccine coverage during a school-based hepatitis B immunization program. Can J Public Health. 1998;89(2):98-101.

31. Nelson JC et al. Compliance with multiple-dose vaccine schedules among older children, adolescents, and adults: results from a Vaccine Safety Datalink Study. (Special Issue: Influenza preparedness and response.). Am J Public Health. 2009;99(Supplement 2):S389-97.

32. Centres for Disease Control and Prevention. Meningococcal: who needs to be vaccinated? http://www.cdc.gov/vaccines/vpd-vac/mening/whovaccinate.htm. 2015 [cited January 2015].

33. Smith LM et al. Factors associated with initiation and completion of the quadrivalent human papillomavirus vaccine series in an ontario cohort of grade 8 girls. BMC Public Health. 2011;11.

34. Lions C, Pulcini C, Verger P. Papillomavirus vaccine coverage and its determinants in South-Eastern France. Med Mal Infect. 2013:43(5):195-201.

35. Macdonald $V$ et al. Predictors of completion of a hepatitis $B$ vaccination schedule in attendees at a primary health care centre. Sex Health. 2007;4(1): 27-30.

36. Chao $\mathrm{C}$ et al. Correlates for completion of 3-dose regimen of HPV vaccine in female members of a managed care organization. Mayo Clin Proc. 2009; 84(10):864-70

37. Schluterman $\mathrm{NH}$ et al. Human papillomavirus (HPV) vaccine uptake and completion at an urban hospital. Vaccine. 2011;29(21):3767-72.

38. Perkins RB et al. Correlates of human papillomavirus vaccination rates in low-income, minority adolescents: a multicenter study. J Womens Health. 2012;21(8):813-20.

39. Dorell CG et al. Human papillomavirus vaccination series initiation and completion, 2008-2009.[Erratum appears in Pediatrics. 2012 Jul;130(1):166-8 Note: Dosage error in article text]. Pediatrics. 2011;128(5):830-9.

40. Neubrand TPL et al. Factors associated with completion of the human papillomavirus vaccine series. Clin Pediatr. 2009;48(9):966-9.

41. Verdenius I et al. Predictors of three dose on-time compliance with HPV4 vaccination in a disadvantaged, underserved, safety net population in the US midwest. PLoS One. 2013:8:8.

42. Widdice LE et al. Adherence to the HPV vaccine dosing intervals and factors associated with completion of 3 doses. Pediatrics. 2011;127(1):77-84

43. Tan $W$ et al. The HPV vaccine: are dosing recommendations being followed? Vaccine. 2011;29(14):2548-54

44. Niccolai LM, Mehta NR, Hadler JL. Racial/ethnic and poverty disparities in human papillomavirus vaccination completion. Am J Prev Med. 2011;41(4): 428-33.

45. Reiter PL, Katz ML, Paskett ED. Correlates of HPV vaccination among adolescent females from Appalachia and reasons why their parents do not intend to vaccinate. Vaccine. 2013;31(31):3121-5.

46. Dempsey A et al. Worsening disparities in HPV vaccine utilization among 19-26 year old women. Vaccine. 2011;29(3):528-34
47. Middleman $A B$ et al. Predictors of time to completion of the hepatitis $B$ vaccination series among adolescents. J Adolesc Health. 1999;25(5):323-7.

48. Harper DM et al. Quantifying clinical HPV4 dose inefficiencies in a safety net population. PLoS One. 2013:8:11.

49. Moss $J \mathrm{~L}$ et al. Organizational correlates of adolescent immunization: Findings of a state-wide study of primary care clinics in North Carolina. Vaccine. 2013;31(40):4436-41.

50. Laz TH, Rahman M, Berenson AB. An update on human papillomavirus vaccine uptake among 11-17 year old girls in the United States: National Health Interview Survey, 2010. Vaccine. 2012;30(24):3534-40.

51. Sakou II et al. Vaccination coverage among adolescents and risk factors associated with incomplete immunization. Eur J Pediatr. 2011;170(11):1419-26.

52. Brotherton JML et al. Human papillomavirus vaccine coverage among female Australian adolescents: success of the school-based approach. Med J Aust. 2013:199(9):614-7.

53. Gold R et al. Completion and timing of the three-dose human papillomavirus vaccine series among adolescents attending school-based health centers in Oregon. Prev Med. 2011;52(6):456-8.

54. Chao $\mathrm{C}$ et al. Papanicolaou screening behavior in mothers and human papillomavirus vaccine uptake in adolescent girls. Am J Public Health. 2009; 99(6):1137-42.

55. Gold R, Naleway A, Riedlinger K. Factors predicting completion of the human papillomavirus vaccine series. J Adolesc Health. 2013;52(4):427-32.

56. Markovitz AR et al. Association between maternal preventive care utilization and adolescent vaccination: it's not just about Pap testing. J Pediatr Adolesc Gynecol. 2014;27(1):29-36.

57. Chao $\mathrm{C}$ et al. Correlates for human papillomavirus vaccination of adolescent girls and young women in a managed care organization. Am J Epidemiol. 2010;171(3):357-67.

58. Musto $\mathrm{R}$ et al. Social equity in human papillomavirus vaccination: a natural experiment in Calgary Canada. BMC Public Health. 2013;13.

59. Sinka $\mathrm{K}$ et al. Achieving high and equitable coverage of adolescent HPV vaccine in Scotland. J Epidemiol Community Health. 2014;68(1):57-63.

60. Bertaut A et al. HPV vaccination coverage in French girls attending middle and high schools: a declarative cross sectional study in the department of Cote d'Or. Eur J Obstet Gynecol Reprod Biol. 2013;170(2):526-32.

61. Reiter PL et al. How much will it hurt? HPV vaccine side effects and influence on completion of the three-dose regimen. Vaccine. 2009;27(49):6840-4.

62. Kouyoumdjian FG, Bailowitz A. Completion of the human papillomavirus vaccine series in females attending an urban immunization clinic. Pediatr Infect Dis J. 2011:30(8):718-9.

63. Cleves MA. Hepatitis B vaccine compliance in inner city youth. J Adolesc Health. 1998:22(2):148.

64. Bednarczyk RA et al. Human papillomavirus vaccine uptake and barriers: association with perceived risk, actual risk and race/ethnicity among female students at a New York State university, 2010. Vaccine. 2011:29(17):3138-43.

65. Brotherton JM, Mullins RM. Will vaccinated women attend cervical screening? A population based survey of human papillomavirus vaccination and cervical screening among young women in Victoria, Australia. Cancer Epidemiol. 2012;36(3):298-302.

66. Monnat SM, Wallington SF. Is there an association between maternal Pap test use and adolescent human papillomavirus vaccination? J Adolesc Health. 2013:52(2):212-8.

67. LaMontagne DS et al. Human papillomavirus vaccine delivery strategies that achieved high coverage in low- and middle-income countries. Bull World Health Organ. 2011;89(11):821-830B.

68. Ladner J et al. Performance of 21 HPV vaccination programs implemented in low and middle-income countries, 2009-2013. BMC Public Health. 2014; 14:670.

69. Ladner J et al. Assessment of eight HPV vaccination programs implemented in lowest income countries. BMC Public Health. 2012:12:370.

70. Tung CS, Middleman AB. An evaluation of school-level factors used in a successful school-based hepatitis B immunization initiative. J Adolesc Health. 2005;37(1):61-8.

71. Lee GM et al. Adolescent immunizations: missed opportunities for prevention. Pediatrics. 2008;122(4):711-7.

72. Wong CA et al. Missed opportunities for adolescent vaccination, 2006-2011. I Adolesc Health. 2013:53(4):492-7.

73. Jacobson Vann JC, Szilagyi P. Patient reminder and recall systems to improve immunization rates. Cochrane Database Syst Rev. 2005; doi: 10. 1002/14651858.CD003941.pub2. 